\title{
Deconfounding the Effects of Congruency and Task Difficulty on Hemispheric Differences in Global/Local Processing
}

\author{
Gregor Volberg and Ronald Hübner
}

Universität Konstanz, Germany

\begin{abstract}
Although it is often assumed that the left and right cerebral hemispheres are specialized for local and global processing, respectively, recent studies show that this difference mainly occurs if the responses to the two levels of a stimulus are conflicting. In the present study we examined whether the favorable effect of response conflicts is caused by the increased task difficulty in this situation. To this end, the response selection for nonconflicting stimuli was complicated by frequently changing the stimulus-response mappings. As a result, the reactions to nonconflicting stimuli were as slow as those to conflicting ones. Nevertheless, hemispheric differences were again restricted to the latter situation. This shows that increased task difficulty can not explain the modulating effect of response conflicts. The results support the alternative hypothesis that different representations are needed for the response selection for nonconflicting and conflicting stimuli, and that the hemispheres differ only with respect to the latter.
\end{abstract}

Keywords: global processing, local processing, hemispheric differences, task difficulty, variable mapping

Although it is widely assumed that the local and global levels of hierarchical stimuli are preferentially processed in the left and right cerebral hemispheres (LH/RH), respectively, the empirical support for this hypothesis is still rather weak. In a typical response-time paradigm, compound letters similar to those in Figure 1 (Navon, 1977) are either presented to the left or to the right visual field (LVF/RVF) and a quick response to a given level is required. Because the letters are projected to the opposite hemisphere in each case, it is expected that responses to the local and global levels are faster for RVF-stimuli and LVF-stimuli, respectively. However, corresponding visual-field (VF) effects were observed only in roughly half of the existing response-time studies (for overview articles see Van Kleeck, 1989; Yovel, Yovel, \& Levy, 2001). Similarly inconclusive results are reported in neuropsychological investigations (Polster \& Rapcsak, 1994; Robertson \& Lamb, 1991; Schatz, Craft, Koby, \& DeBaun, 2004), neuroimaging studies (Fink et al., 1996; Heinze, Hinrichs, Scholz, Burchert, \& Mangun, 1998), and in studies measuring event-related brain potentials (ERPs; e.g., Han, Fan, Chen, \& Zhuo, 1999; Volberg \& Hübner, 2004).

Taken together, the existing results suggest that hemispheric differences with respect to global and local processing are not a general and robust phenomenon. Rather, they seem to depend on certain experimental conditions. For instance, hemispheric differences are more prominent if the saliency of the global and local levels is equal (Yovel et al., 2001), if the task requires attention to both levels (Heinze et al., 1998), and if the target level is unpredictable (Hübner \& Malinowski, 2002). In a further attempt to isolate favorable factors, Hübner and Malinowski (2002) investigated the effect of response conflicts on hemispheric differences. They found VF-effects only for incongruent stimuli, i.e., for stimuli where the information at the nontarget level activated a different response than that on the target level. When the information at both levels was congruent there were no VF-effects (see also Van Kleeck, 1989). Furthermore, Hübner and Malinowski found that varying the size of the response conflict did not produce a respective modulation of the VF-effects. That is, response conflicts had a qualitative effect on hemispheric differences in that they produced VF-effects irrespective of their size.

In a similar manner, response conflicts also determine the occurrence of hemispheric ERP differences. In a recent study we presented congruent and incongruent stimuli to the LVF or to the RVF and collected behavioral data as well as brain responses (Volberg \& Hübner, 2004). As a result, hemispheric ERP asymmetries and the expected behavioral VF-effects were found. However, in both measures, hemispheric differences again mainly occurred in the incongruent case (see also Malinowski, Hübner, Keil, \& Gruber, 2002).

In considering these results, the question arises as to how 

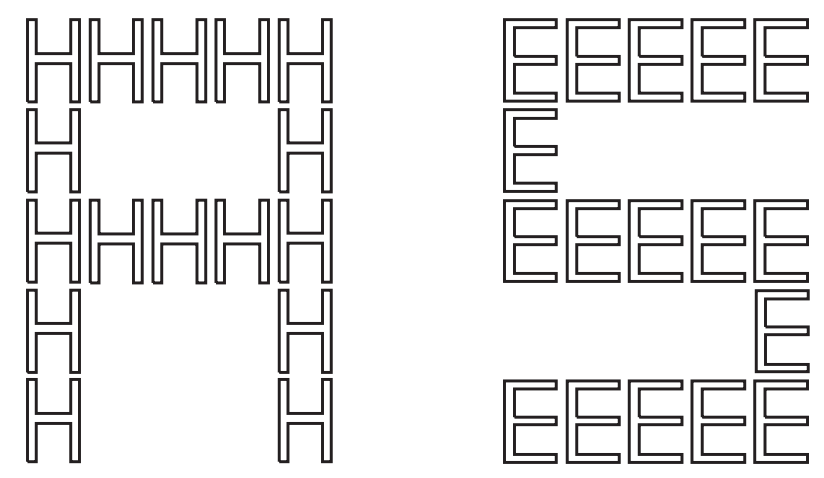

Figure 1. Two examples of stimuli used in the present study.

response conflicts can modulate the occurrence of hemispheric differences. Hübner and Malinowski (2002) offered a reasonable answer. They proposed that at an early stage of processing the global and local letters are represented independently of their respective level, and that the hemispheres do not differ with respect to these representations. This hypothesis has been further elaborated and empirically supported in a recent paper (Hübner \& Volberg, 2005). It follows from this account that, when response selection can be based on early stimulus representations, no hemispheric differences are observed. This is what usually happens with congruent stimuli. Since all the information activates the same response, it is not necessary to consider any level information. On the other hand, when the letter at the nontarget level activates a different response than that at the target level, response selection requires a more elaborated stimulus representation where the letters are integrated with their respective levels. In this case, there are hemispheric differences. Therefore, Hübner and coworkers (2002, 2005) suggested that the hemispheres differ with respect to their capacity for integrating forms with their respective stimulus level. Specifically, the LH has a relative advantage compared to the $\mathrm{RH}$ for integrating forms with the local level, whereas the $\mathrm{RH}$ is more proficient than the LH at integrating forms with the global level.

Although this integration hypothesis provides a sound explanation for the favorable effect of response conflicts on hemispheric differences, there is one difficulty with this interpretation for response-time data. Usually, incongruent stimuli produce slower responses and more errors than congruent ones (Eriksen \& Eriksen, 1974). For example, responses to incongruent stimuli were $28 \mathrm{~ms}$ slower than those to congruent stimuli in Volberg and Hübner (2004), and $42 \mathrm{~ms}$ slower in the Hübner and Malinowski study (2002, Experiment 2). Therefore, an alternative explanation of the data could be that VF-effects do not depend on congruency but rather on task difficulty. In other words, it could be argued that hemispheric differences are restricted to incongruent stimuli simply because this is the more difficult condition.

The possibility that task difficulty might be a relevant factor has already been pointed out by Hübner and Malinowski (2002). However, they argued that this hypothesis was too general for explaining the presence of VF-effects in hierarchical processing and, therefore, rejected it. However, even if task difficulty does not explain which mechanisms actually produce the hemispheric difference, it nonetheless provides an alternative account of why VF-effects depend on response conflicts. It is conceivable that hemispheric differences occur only if the task is highly demanding. In easy situations, the respectively weaker hemisphere may be as effective as the stronger one.

Indeed, there is some evidence supporting such an interpretation. For example, Banich and Belger (1990) presented two single letters either to the same or to different hemispheres and varied the complexity of the associated task (letter matching vs. semantic classification). It turned out that in the easier condition the responses were faster when both letters were presented to the same hemisphere. Contrarily, in the more difficult situation they were faster when the letters were distributed across hemispheres. This suggests that, even for obviously lateralized tasks like verbal decisions, it is often more efficient to process the information in the hemisphere where it is first available. However, if the task is difficult, it is favorable to distribute computations between the hemispheres (see also Banich \& Weissman, 2000; Belger \& Banich, 1998). This processing rule could also be relevant for hemispheric differences in hierarchical processing. Since response conflicts generally increase the task difficulty they should also increase the probability that local and global information is directed to and processed within the specialized hemisphere. As a result, VF-effects should show up only in such conditions.

Irrespective of whether this alternative account is valid, the problem persists that two factors were confounded in previous research on VF-effects in hierarchical processing. On the one hand, the congruency of the global and local information was manipulated. On the other hand, as a sideeffect, the task difficulty also covaried. As long as this confound exists, the results of previous studies investigating the role of response conflicts (e.g., Hübner \& Malinowski, 2002; Malinowski et al., 2002; Van Kleeck, 1989; Volberg \& Hübner, 2004) can hardly be interpreted in a unique way. Therefore, the aim of the present experiment was to show the positive effects of incongruent stimuli on VF-effects not confounded by task difficulty. The idea was to equalize task difficulty for congruent and incongruent stimuli, and then compare the VF-effects. If task difficulty is the relevant factor, then the VF-effects should not differ. However, if congruency is crucial, then the VF-effects should still be restricted to the incongruent condition.

How can task difficulty be balanced between the congruent and incongruent conditions? According to the integration account, the conditions differ with respect to the mode of response selection (see Hübner \& Malinowski, 2002; Hübner \& Volberg, 2005). In the congruent condition the forms at the levels activate the same response so it can be selected in a more automatic (and, hence, in a faster) 
mode. By contrast, in the incongruent situation the available information is ambiguous, so the subjects must select the response in a more controlled (and, hence, in a slower) mode (Shiffrin \& Schneider, 1984). Thus, even with extensive practice, it is unlikely that the incongruent condition could be made as easy as the congruent one. Fortunately, it is easier to make the congruent condition more difficult. If the mapping of letters onto responses is frequently varied within the same experiment, then automatic responses cannot be established. Rather, subjects have to rely on a controlled memory search for retrieving the appropriate response, which should substantially increase the response times for congruent stimuli compared to a constant mapping (Schneider \& Shiffrin, 1977; Shiffrin \& Schneider, 1977). In contrast, a varied mapping should hardly affect the performance for incongruent stimuli, since response selection generally proceeds in a controlled mode for these stimuli. Accordingly, the difference between the response times for the two stimulus types should be diminished.

Nevertheless, even if both stimulus types are processed in a controlled mode, they still differ with respect to congruency. Therefore, the crucial difference with respect to VF-effects remains. If the letters are incongruent, it is necessary to combine the letters and their levels in order to select the appropriate response. In contrast, for congruent letters such a combination would not be necessary. This is true even if the responses are retrieved in a controlled (i.e., slow) manner. The fact that the processing mode (automatic vs. controlled) can be varied independently of congruency (congruent vs. incongruent) makes varied mapping an ideal method for deconfounding the effects of task difficulty and congruency. Therefore, it was utilized in the present study.

Congruent and incongruent stimuli were presented to the LVF or to the RVF, and a quick response to a specific level was required. The stimuli contained letters with a variable mapping, so that automatic responses should be prevented. Furthermore, the number of trials within each block was small $(n=32)$ in order to hinder the subjects from learning. Accordingly, we expected that performance should be similar in the two congruency conditions. Consequently, if task difficulty is the crucial factor for hemispheric differences, then the VF-effects should not differ for congruent and incongruent stimuli. On the other hand, if congruency is the important factor, then VF-effects should again occur only for incongruent stimuli.

\section{Method}

\section{Subjects}

The subjects were 16 students of the University of Konstanz (10 female, 6 male, mean age 25.3 years). All of them were right-handed by self-report and had normal or corrected-to-normal vision. In return for their participation, the students either received course credits or a fee of $5 €$ per hour.

\section{Apparatus}

The stimuli were generated on a personal computer and presented on a 21-inch color monitor (Sony 500PS). The screen resolution was $1280 \times 1024$ pixels at a vertical refresh rate of $75 \mathrm{~Hz}$. Responses were given on a two-button device, and were registered by the same computer that was used to control the stimulus presentation.

\section{Stimuli}

Stimuli were hierarchical letters constructed from identical capitals in a $5 \times 5$ grid (Figure 1). The letters $A$ and $E$ were combined with the letters $S$ and $H$, respectively, resulting in eight different stimuli: $A-s$ ("global $A$ - local $S$ "), $A-h$, $E-s, E-h, S-a, S-e, H-a$, and $H-e$. For half of the subjects, the letters $A$ and $E$ were mapped onto alternative response buttons in a constant manner, whereas the mapping of letters $S$ and $H$ to the left and right response button was reversed after each block. For the other half, the mapping of $S$ and $H$ was constant and that of $A$ and $E$ was variable. Thus, each stimulus contained one letter with a constant and one with a variable mapping. The reason for varying the mapping of only one out of the two letters is that the task becomes extremely difficult if the mapping of both letters is varied at the same time. In a few test runs with this procedure, the participants produced unacceptably high error rates. Therefore, variable mapping was restricted to one letter. This policy ensured that the information contained within one stimulus was always ambivalent concerning the response. Thus, with respect to the whole stimulus the mapping was never constant, so that automatic responses were not possible.

Half of the stimuli were congruent, whereas the other half were incongruent. The size of the global form was $3.3^{\circ}$ of visual angle horizontally and $4.5^{\circ}$ vertically, and that of the local elements was $0.6^{\circ} \times 0.7^{\circ}$. The stimuli were presented to the LVF or to the RVF, where the inner edge of the stimulus was vertically aligned to the midline of the screen. Accordingly, the eccentricity was $1.65^{\circ}$ of visual angle, measured from fixation to the center of the stimulus. A small eccentricity was chosen because this is a favorable condition for hemispheric differences in global and local processing (van Kleeck, 1989).

\section{Procedure}

A trial began with a $300 \mathrm{~ms}$ presentation of a cue at the center of the screen. The letters $G$ and $L$ were used to indicate a global or local target level, respectively, and at the same time served as a fixation mark. The participants were 


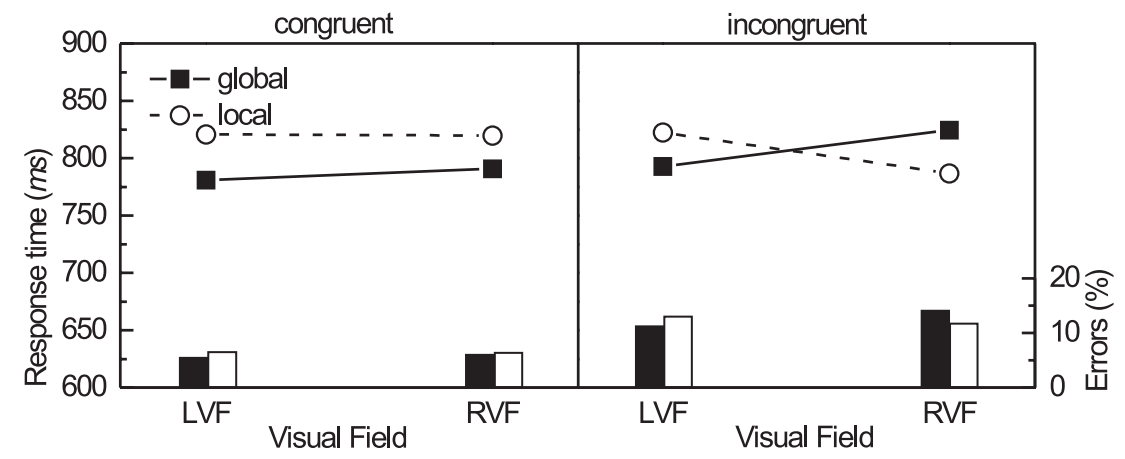

Figure 2. Data showing the three-way interaction between the factors Target Level, Visual Field, and Congruency. Notice that the overall response times to congruent and incongruent stimuli do not differ.

instructed to maintain the central fixation throughout the experiment. After an interval of $300 \mathrm{~ms}$ the stimulus was presented for $93 \mathrm{~ms}$ in the LVF or RVF. The task was to identify the letter on the cued level as fast and as accurate as possible by pressing the corresponding response button. Half of the subjects responded with the index and middle finger of the left hand, and the other half used the right hand. Wrong responses were signaled by a short tone.

Three factors were varied in the present experiment, all of which were randomized: Target Level (global and local), Visual Field (LVF and RVF), and Congruency (congruent and incongruent). After four training blocks, the subjects performed 12 experimental blocks with 32 trials at a time. Accordingly, each of the 8 conditions was covered by 48 observations per subject. The data were collected within one single session of approximately $1 \mathrm{~h}$.

\section{Results}

Response latencies for correct responses were entered into a three-factorial analysis of variance (ANOVA) with repeated measures on all factors. The same ANOVA was computed for the error rates as dependent variable. Post hoc comparisons were performed by means of planned contrasts.

\section{Response Latencies}

Main effects with respect to response latencies were not observed. It is especially important to note that the response times to congruent and incongruent stimuli were almost identical (803 and $807 \mathrm{~ms}$, respectively). Accordingly, the difference was far from significant, $F(1,15)=.10, p=.75$.

With respect to VF-effects, there was a two-way interaction between the factors Target Level and Visual Field, $F(1,15)=8.61, p<.05$, which was qualified by a three-way interaction between Target Level, Visual Field, and Congruency, $F(1,15)=5.16, p<.05$. It was decomposed by computing VF-effects separately for the congruent and for the incongruent condition. The results are depicted in Figure 2. As one can see, VF-effects occurred only for incon- gruent stimuli, $F(1,15)=11.84, p<.01$. In this condition, responses to the local level were faster for RVF-stimuli (787 ms) compared to LVF-stimuli $(822 \mathrm{~ms})$, whereas forms on the global level were processed faster in LVFstimuli (793 ms) compared to stimuli in the RVF (824 ms). The corresponding differences in the congruent condition were not reliable, $F(1,15)=0.41, p=.53$.

\section{Error Rates}

With error rates as dependent variable, the ANOVAs revealed a main effect for the factor Congruency, $F(1,15)=$ $57.35, p<.001$. It showed that responses were more accurate in the congruent $(6.35 \%$ errors $)$ compared to the incongruent condition $(12.63 \%)$. Further significant results were not observed.

\section{Discussion}

Results from response-time experiments (Hübner \& Malinowski, 2002; Van Kleeck, 1989) and ERP studies (Malinowski et al., 2002; Volberg \& Hübner, 2004) suggest that hemispheric differences for global and local processing mainly occur if the responses to the levels are conflicting. Unfortunately, two factors were confounded in these studies: the congruency of the letters at the levels, and task difficulty. The aim of the present study was to deconfound these factors. To this end, the difficulty of the congruent and incongruent conditions was balanced by means of a varied mapping procedure. If task difficulty was the crucial variable, then VF-effects should not differ between the two congruency conditions. On the other hand, if congruency is important, then they should again be restricted to the incongruent case.

As can clearly be seen in Figure 2, congruency is the important factor. Although the difficulty of the congruent and the incongruent conditions was nearly identical, VFeffects were restricted to the latter case. With congruent stimuli there were no hemispheric differences. Thus, the favorable effect of response conflicts that has been ob- 
served in earlier studies can not be explained by the increased task difficulty in this situation. Rather, an incongruency between the levels seems to be crucial. The data are, therefore, fully supportive of the integration hypothesis (Hübner \& Malinowski, 2002; Hübner \& Volberg, 2005). Because the hemispheres differ in their efficiency for level and form integration, and because such integration is necessary only in the incongruent condition, VF-effects are restricted to that situation.

One problem might arise from our assumption that, as long as response times do not differ between congruent and incongruent stimuli, the task difficulty is equal in the two conditions. Specifically, it is possible that neural activities associated with the congruent and incongruent conditions are different even though response times do not differ. On the other hand, response time measures usually covary with indices of neural activity. For example, in the initial ERP study on global and local processing, response times to the global and local levels closely matched the latencies of the temporal N2 and the parietal P3 components (Heinze \& Münte, 1993). Also, frontal N2 latencies covary with response latencies to congruent and incongruent stimuli (Han, Fan, Chen, \& Zhuo, 1997; Han et al., 1999; Han, He, Yund, \& Woods, 2001). By considering that response time differences rely on differences in neural activity, for the present purpose it seems sufficient to define task difficulty by means of response speed alone.

Another possible objection is that VF-effects occur whenever just one of the two letters in a hierarchical stimulus leads to a correct response, regardless of whether the second letter is conflicting. Specifically, hemispheric differences could also occur if the irrelevant letter is neutral with respect to the response. Such a result would invalidate the integration approach, because no integration is necessary for response selection in this situation. This explanation cannot be ruled out by means of the present data. However, it was adequately addressed in a former study (Hübner \& Malinowski, 2002). In different blocks of trials, congruent stimuli were either intermixed with incongruent stimuli or with neutral ones. It turned out that VF-effects were prominent only in the incongruent condition. Thus, the above mentioned objection can be declined.

A related objection is that, in the absence of a main effect of congruency, there is no behavioral evidence of response conflict in the present study. Thus, one could also conclude that VF-effects can occur without response conflicts. At first glance, this seems to contradict the integration hypothesis. However, the critical point for VF-effects is the requirement to integrate levels with their letters, and not response conflict as such. Thus, if there is more than one stimulus-response mapping, as in the present case, then an integration of levels and their letters is needed for response selection, irrespective of the size or presence of response conflicts. This claim is also supported by the study of Hübner and Malinowski (2002) where, in the incongruent situation, the size of VF-effects did not covary with the magnitude of response conflict generated by those stimuli.
A striking result of this study is that, although the response times to congruent and incongruent stimuli were nearly identical, the error rates still differed in these situations. Indeed, they were about $6 \%$ and $13 \%$, respectively. This seems to indicate that the task difficulty also differed between the conditions. However, we could show empirically as well as by means of a computational model (Hübner \& Volberg, 2005) that the differences in error rates are a direct consequence of the integration mechanism. Because the letters can be coded independently of their respective levels, subjects often confuse the letter at the nontarget level with that at the target level. For congruent stimuli, such confusion errors do not lead to erroneous responses as both letters require the same answer anyway. In contrast, for incongruent stimuli confusion errors produce incorrect answers. Thus, because confusion errors remain undetected in congruent situations, the error rates are lower compared to incongruent or neutral situations, even if the task difficulty is equal. For example, the error rate for congruent stimuli was $3.63 \%$ compared to $7.65 \%$ for neutral ones in Hübner \& Malinowski, 2002 (Experiment $3)$. This proportion is very similar to the difference observed in the present study. By considering this explanation, it is unlikely that the observed difference between error rates to congruent and incongruent stimuli reflects a substantial difference in task difficulty that could account for the differential VF-effects.

To sum up, the results of the present study show that the favorable effect of congruency and the related response conflicts on VF-effects is not caused by the (usually) increased task difficulty in that situation. Rather, incongruency between the levels is crucial. Because an integration of level and form is necessary for response selection in this situation, the data fit well with the hypothesis that the hemispheres differ in this integration process.

\section{Acknowledgments}

This research was supported by a grant from the Deutsche Forschungsgemeinschaft (DFG) to the second author as part of a research group (Hu 432/7-4). We thank Franka Glöckner for the data acquisition, and Tobias Studer for helpful comments on this paper.

\section{References}

Banich, M.T., \& Belger, A. (1990). Interhemispheric interaction: How do the hemispheres divide and conquer a task? Cortex, 26, 77-94.

Banich, M.T., \& Weissman, D.H. (2000). One of twenty questions for the twenty-first century: How do brain regions interact and integrate information? Brain and Cognition, 42, 29-32.

Belger, A., \& Banich, M.T. (1998). Costs and benefits of integrat- 
ing information between the cerebral hemispheres: A computational perspective. Neuropsychology, 12, 380-398.

Eriksen, B.A., \& Eriksen, C.W. (1974). Effects of noise letters upon the identification of a target letter in a nonsearch task. Perception and Psychophysics, 16, 143-149.

Fink, G.R., Halligan, P.W., Marshall, J.C., Frith, C.D., Frackowiak, R.S., \& Dolan, R.J. (1996). Where in the brain does visual attention select the forest and the trees? Nature, 382, 626-628.

Han, S., Fan, S., Chen, L., \& Zhuo, Y. (1997). On the different processing of wholes and parts: A psychophysiological analysis. Journal of Cognitive Neuroscience, 9, 687-698.

Han, S., Fan, S., Chen, L., \& Zhuo, Y. (1999). Modulation of brain activities by hierarchical processing: A high-density ERP study. Brain Topography, 11, 171-183.

Han, S., He, X., Yund, E.W., \& Woods, D.L. (2001). Attentional selection in the processing of hierarchical patterns: An ERP study. Biological Psychology, 56, 113-130.

Heinze, H.J., Hinrichs, M., Scholz, M., Burchert, W., \& Mangun, G.R. (1998). Neural mechanisms of global and local processing: A combined PET and ERP study. Journal of Cognitive Neuroscience, 10, 485-498.

Heinze, H.J., \& Münte, T.F. (1993). Electrophysiological correlates of hierarchical stimulus processing: Dissociation between onset and later stages of global and local target processing. Neuropsychologia, 31, 841-852.

Hübner, R., \& Malinowski, P. (2002). The effect of response competition on functional hemispheric asymmetries for global/local processing. Perception and Psychophysics, 64, 1290-1300.

Hübner, R., \& Volberg, G. (2005). The integration of object levels and their content: A theory of global/local processing and related hemispheric differences. Journal of Experimental Psychology: Human Perception and Performance, 31, 520-541.

Malinowski, P., Hübner, R., Keil, A., \& Gruber, T. (2002). The influence of response competition on cerebral asymmetries for processing hierarchical stimuli revealed by ERP recordings. Experimental Brain Research, 144, 136-139.

Navon, D. (1977). Forest before the trees: The precedence of global features in visual perception. Cognitive Psychology, 9, 353-393.

Polster, M.R., \& Rapcsak, S.Z. (1994). Hierarchical stimuli and hemispheric specialization: Two case studies. Cortex, 30, 487497.
Robertson, L.C., \& Lamb, M.R. (1991). Neuropsychological contributions to theories of part/whole organization. Cognitive Psychology, 23, 299-330.

Schatz, J., Craft, S., Koby, M., \& DeBaun, M.R. (2004). Asymmetries in visual-spatial processing following childhood stroke. Neuropsychology, 18, 340-352.

Schneider, W., \& Shiffrin, R.M. (1977). Controlled and automatic human information processing: I. Detection, search, and attention. Psychological Review, 84, 1-66.

Shiffrin, R.M., \& Schneider, W. (1977). Controlled and automatic human information processing: II. Perceptual learning, automatic attending, and a general theory. Psychological Review, 84, 127-190.

Shiffrin, R.M., \& Schneider, W. (1984). Automatic and controlled processing revisited. Psychological Review, 91, 269-276.

Van Kleeck, M.H. (1989). Hemispheric differences in global versus local processing of hierarchical visual stimuli by normal subjects: New data and a meta-analysis of previous studies. Neuropsychologia, 27, 1165-1178.

Volberg, G., \& Hübner, R. (2004). On the role of response conflicts and stimulus position for hemispheric differences in global/local processing: An ERP study. Neuropsychologia, 42, 1805-1813.

Yovel, G., Yovel, I., \& Levy, J. (2001). Hemispheric asymmetries for global and local visual perception: Effects of stimulus and task factors. Journal of Experimental Psychology: Human Perception and Performance, 27, 1369-1385.

Received September 8, 2005

Revision received January 11, 2006

Accepted March 6, 2006

Gregor Volberg

Universität Regensburg

Institut für Experimentelle Psychologie

Universitätsstr. 31

D-93053 Regensburg

Germany

E-mail gregor.volberg@psychologie.uni-regensburg.de 